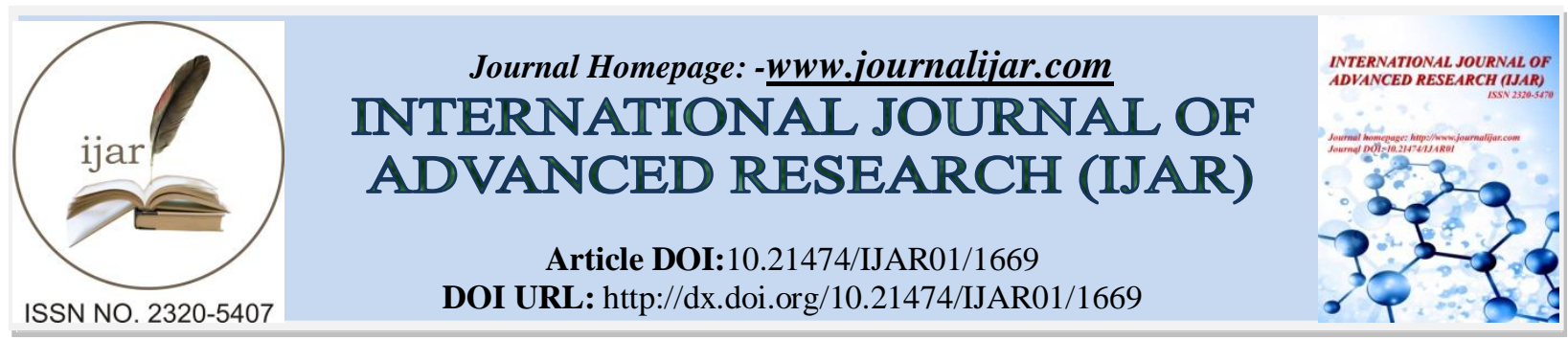

RESEARCH ARTICLE

\title{
POLLEN MORPHOLOGICAL STUDIES ON TWO SOLANACEOUS GENERA: BRUGMANSIA PERS. AND DATURA L.
}

Dhanya $\mathbf{C}^{\mathbf{1}}$ and Devipriya $\mathrm{V}^{\mathbf{2}}$.

1. Post Graduate \& Research Department of Botany, Sree Narayana College, Kollam, Kerala.

2. Department of Botany, Sree Narayana College, Chempazhanthy, Thiruvananthapuram, Kerala.

\section{Manuscript Info}

Manuscript History

Received: 13 July 2016

Final Accepted: 12 August 2016

Published: September 2016

Key words:-

Pollen morphology, Brugmansia,

Datura, mural subunits, microscabrae

\section{Abstract}

The exomorphological features of the pollen grains of five taxa of DaturaL. and six taxa of BrugmanisaPers. (Solanaceae) from Kerala and Tamil Nadu, India, have been studiedin detail. The two genera are closely related withBrugmansiabeing formerly included under Datura and later given a generic status. The pollen of both plant groups are also similar being 3-zonocolporate,large or medium andstriateor striato-reticulate, occasionally rugulate. The transverse or longitudinal alignment of the mural subunits in the exine has been considered to be the major distinguishing feature between pollen of the two genera. But in the present study, longitudinal and/or diagonal orientation of mural subunits has been observed in two varieties of $B$. sanguinea. Hence mural subunit alignment cannot be depended upon as a unique distinguishing character between pollen of the two genera. Although microscabrae were reported earlier from some members in both genera,adequate attention seems to be lacking with regard to these bodies. In the present study,microscabrae were observed in all the eleven members studied and showed distinction between the two genera, being large, nodular and dense in single or more rows in Brugmansia and small, granular, sparse and irregularly scattered in members of Datura. On the basis of the present observations, two pollen types are proposed here for the two genera studied viz. Brugmansia -type and Datura -type.

Copy Right, IJAR, 2016,. All rights reserved.

\section{Introduction:-}

Palynology has moved beyond the realms of classical exine characteristics relating to the aperture, exine ornamentation, exine strata, pollen size and pollen shape to in-depth microstructural analysis of the delicate patterns of exine surface ornamentation employing highly sophisticated tools such as the Scanning Electron Microscope. The 'micro palynological markers' so unearthed are often characteristic to particular plant groups, and are now increasingly depended upon as supplementary tools in deciphering the complex patterns of taxonomic interrelationships and species delimitations.

Datura L. and BrugmansiaPers. belong to the deadly nightshade family Solanaceae, comprising of over 94 genera and 2950 species worldwide (Mabberly, 2005; Shultes and Raffauf, 1991). Datura L., is native to Northern 
America, and comprises of 10-12 species distributed in the tropical and temperate regions of the world, of which four have been reported from South India (Sasidharan, 2004). The genus includes several medicinal plants and some weedy species. BrugmansiaPers. (formerly included under Datura and later given a generic status by Lockwood, 1973), is native to South America and includes six to nine species of small ornamental trees, of which only one has so far been reported from South India.

The earlier palynological literaturelargely focuses on the economically more valuable genus - Datura, and includes the study on the pollen grains of eleven taxa of Chinese Datura by Guang-Fang et al. (1985), Daturastramoniumfrom Jordhanby Al-Quran (2004),three species of Datura from Pakistanby PerveenandQaiser (2007)and Datura metel from Nigeria (Adedeji and Akinniyi, 2015). Although Persoonet al. (1999) compared the pollen morphology of five species of Brugmansia and eight species of Datura from America,palynological studies on the taxa recorded from India are scanty. The present study focuses on the comparative evaluation of the fine exomorphological features of the pollen of six taxa of Brugmanisaand five taxa of Daturafrom Kerala and Tamil Nadu, India using Light Microscopy and Scanning Electron Microscopy.

\section{Materials and Methods:-}

The details regarding the taxa selected for the present study from Kerala and Tamil Nadu are furnished below in Table -1 .

Table 1:- Details of thetaxa of BrugmansiaPers. andDaturaL. collected for the present study form Kerala and Tamil Nadu

\begin{tabular}{|c|l|l|c|}
\hline Sl. No & \multicolumn{1}{|c|}{ Name of species } & \multicolumn{1}{c|}{ Locality } & \multicolumn{1}{|c|}{ Voucher No. } \\
\hline 1 & BrugmansiaxcandidaPers. 'Charleston' & Kodaikanal, Tamilnadu & S015 SNCH \\
\hline 2 & Brugmansiax cubensis (V.R.Fuentes) V.R.Fuentes 'Dr.Seuss' & Kodaikanal, Tamilnadu & S016 SNCH \\
\hline 3 & Brugmansiasanguinea(Ruiz \&Pav.) D. Don 'MishaTora' & Ootacamund, Tamilnadu & S013 SNCH \\
\hline 4 & Brugmansia sanguinea (Ruiz \&Pav.) D. Don 'Oroverde' & Ootacamund, Tamilnadu & S014 SNCH \\
\hline 5 & $\begin{array}{l}\text { Brugmansiasuaveolens(Humb.\&Bonpl.exWilld.) Bercht\&J. } \\
\text { Presl. 'Remembrance' }\end{array}$ & Kottayam, Kerala & S012 SNCH \\
\hline 6 & $\begin{array}{l}\text { Brugmansiasuaveolens(Humb.\&Bonpl.exWilld.) Bercht\&J. } \\
\text { Presl. 'Valley White' }\end{array}$ & Kodaikanal, Tamilnadu & S018 SNCH \\
\hline 7 & Datura innoxia Mill. & Coimbatore, Tamilnadu & S019 SNCH \\
\hline 8 & Datura metel L.var. fastuosa (L.)Saff. & $\begin{array}{l}\text { Thiruvananthapuram, } \\
\text { Kerala }\end{array}$ & S020 SNCH \\
\hline 9 & Datura metel L.var. metel & Kollam, Kerala & S010 SNCH \\
\hline 10 & Datura metel L.var.rubraBernh. & Palakkad, Kerala & S011 SNCH \\
\hline 11 & Datura stramoniumL. & Ootacamund, Tamilnadu & S017 SNCH \\
\hline
\end{tabular}

Polliniferous materials were collected from live plants and fixed in glacial acetic acid, followed by acetolysisafterErdtman (1952) and Nair (1970). Morphological features relating to the pollen aperture, exine ornamentation, pollen size and shape were studied from LM and SEM observations. Pollen measurements were made using an ocular micrometer, from a random sample of 30 pollen grains from each taxon studied. The terminologies suggested by Punt etal. (1994) have been usedto describe aperture types and exine ornamentation pattern.

\section{Results and Discussion:-}

The pollen were 3-zonocolporate and large (B. suaveolens and D.inoxia) or medium in size. Brugmansiapollen were in general medium or large-sized, prolate-spheroidal or oblate-spheroidal to suboblate, 3-zonocolporate and striate (Table-2; Fig. 2), while those of Datura were mostly medium-sized, 3-zonocolporate, striato-reticulate, sometimes rugulateand possessing variable forms (Table-3; Fig. 15). Endocingulum was a common feature in both genera. Acolpate and monocolpate pollen and thin exine were observed in the three varieties of D. metel. The polar outline was rounded with apertures in the obtuse angles, while the equatorial outline was rounded or elliptic, and occasionallyslightly rectangular as in some members of Brugmansia (Fig.1). 
Table 2:- Pollen morphological characters in species of Brugmansia Pers. collected fromKerala and Tamil Nadu

\begin{tabular}{|c|c|c|c|c|c|c|}
\hline Characters & $\begin{array}{l}\text { B. x candida } \\
\text { 'Charleston' }\end{array}$ & $\begin{array}{l}\text { B. cubensis } \\
\text { 'Dr.Seuss' }\end{array}$ & $\begin{array}{l}\text { B. sanguinea } \\
\text { 'MishaTora' }\end{array}$ & $\begin{array}{l}\text { B. sanguinea } \\
\text { 'Oroverde' }\end{array}$ & $\begin{array}{c}\text { B. suaveolens } \\
\text { 'Remembrance' }\end{array}$ & $\begin{array}{l}\text { B. suaveolens } \\
\text { 'Valley } \\
\text { White' }\end{array}$ \\
\hline \multicolumn{7}{|c|}{ Quantitative characters } \\
\hline $\begin{array}{l}\text { Polar diameter - } \\
\mathrm{P}(\mu \mathrm{m})\end{array}$ & $34.20 \pm 2.53$ & $31.20 \pm 3.23$ & $35.40 \pm 1.26$ & $26.40 \pm 3.10$ & $57.00 \pm 4.00$ & $73.20 \pm 6.51$ \\
\hline $\begin{array}{l}\text { Equatorial } \\
\text { diameter. } \mathrm{E} \\
(\mu \mathrm{m})\end{array}$ & $32.40 \pm 2.37$ & $34.80 \pm 1.55$ & $34.80 \pm 3.22$ & $25.20 \pm 1.55$ & $69.00 \pm 5.29$ & $78.00 \pm 5.66$ \\
\hline $\mathrm{P} / \mathrm{E}$ & $1.06 \pm 0.14$ & $0.90 \pm 0.10$ & $1.02 \pm 0.10$ & $1.06 \pm 0.17$ & $0.83 \pm 0.11$ & $0.94 \pm 0.04$ \\
\hline $\begin{array}{l}\text { Exine thickness } \\
(\mu \mathrm{m})\end{array}$ & $3.00 \pm 0.00$ & $3.60 \pm 1.26$ & $3.60 \pm 1.26$ & $3.60 \pm 1.27$ & $5.40 \pm 1.26$ & $5.40 \pm 1.26$ \\
\hline Aperture no. & 3 & 3 & 3 & 3 & 3 & 3 \\
\hline \multicolumn{7}{|c|}{ Qualitative characters } \\
\hline Pollen type & $\begin{array}{l}\text { 3- } \\
\text { zonocolporat } \\
\text { e }\end{array}$ & $\begin{array}{l}\text { 3- } \\
\text { zonocolpora } \\
\text { te }\end{array}$ & $\begin{array}{l}\text { 3- } \\
\text { zonocolporate }\end{array}$ & $\begin{array}{l}\text { 3- } \\
\text { zonocolporate }\end{array}$ & 3-zonocolporate & $\begin{array}{l}\text { 3- } \\
\text { zonocolporate }\end{array}$ \\
\hline $\begin{array}{l}\text { Pollen size- } \\
\text { class }\end{array}$ & Medium & Medium & Medium & Medium & Large & Large \\
\hline $\begin{array}{l}\text { Acolpate / } \\
\text { monocolpate } \\
\text { grain presence }\end{array}$ & Absent & Absent & Absent & Absent & Absent & Absent \\
\hline Polar outline & $\begin{array}{l}\text { Rounded } \\
\text { with } \\
\text { apertures in } \\
\text { the obtuse } \\
\text { angles }\end{array}$ & $\begin{array}{l}\text { Rounded } \\
\text { with } \\
\text { apertures in } \\
\text { the obtuse } \\
\text { angles }\end{array}$ & $\begin{array}{l}\text { Rounded / } \\
\text { slightly trilobed }\end{array}$ & $\begin{array}{l}\text { Rounded with } \\
\text { apertures in } \\
\text { the obtuse } \\
\text { angles }\end{array}$ & $\begin{array}{l}\text { Rounded with } \\
\text { apertures in the } \\
\text { obtuse angles }\end{array}$ & $\begin{array}{l}\text { Rounded with } \\
\text { apertures in } \\
\text { the obtuse } \\
\text { angles }\end{array}$ \\
\hline $\begin{array}{l}\text { Equatorial } \\
\text { outline }\end{array}$ & $\begin{array}{l}\text { Rounded/ } \\
\text { slightly } \\
\text { rectangular }\end{array}$ & $\begin{array}{l}\text { Rounded/ell } \\
\text { iptic }\end{array}$ & $\begin{array}{l}\text { Rounded/ellipti } \\
\text { c/ slightly } \\
\text { rectangular }\end{array}$ & $\begin{array}{l}\text { Rounded/ellipt } \\
\text { ic }\end{array}$ & Rounded/elliptic & $\begin{array}{l}\text { Rounded/ellipt } \\
\text { ic/ slightly } \\
\text { rectangular }\end{array}$ \\
\hline Pollen shape & $\begin{array}{l}\text { Prolate- } \\
\text { spheroidal }\end{array}$ & $\begin{array}{l}\text { Oblate- } \\
\text { spheroidal }\end{array}$ & $\begin{array}{l}\text { Prolate- } \\
\text { spheroidal }\end{array}$ & $\begin{array}{l}\text { Prolate- } \\
\text { spheroidal }\end{array}$ & Suboblate & $\begin{array}{l}\text { Oblate- } \\
\text { spheroidal }\end{array}$ \\
\hline $\begin{array}{l}\text { Exine thickness } \\
\text { in optical CS }\end{array}$ & Thick & Thick & Thick & Thick & Thick/thin & Thick/thin \\
\hline $\begin{array}{l}\text { Endocingulum } \\
\text { presence }\end{array}$ & Present & Present & Present & Present & Present & Present \\
\hline $\begin{array}{l}\text { Exine } \\
\text { ornamentation }\end{array}$ & Striate & Striate & Striate & Striate & Striato-reticulate & $\begin{array}{l}\text { Striato- } \\
\text { reticulate }\end{array}$ \\
\hline $\begin{array}{l}\text { Tectum at } \\
\text { mesocolpium }\end{array}$ & Striate & Striate & Striate & Striate & Striato-reticulate & $\begin{array}{l}\text { Striato- } \\
\text { reticulate }\end{array}$ \\
\hline $\begin{array}{l}\text { Tectum at } \\
\text { apocolpium }\end{array}$ & Striate & Rugulate & Striate & Rugulate & Striato-reticulate & $\begin{array}{l}\text { Striato- } \\
\text { reticulate }\end{array}$ \\
\hline Lumina nature & $\begin{array}{l}\text { Not clearly } \\
\text { visible }\end{array}$ & $\begin{array}{l}\text { Not clearly } \\
\text { visible }\end{array}$ & $\begin{array}{l}\text { Not clearly } \\
\text { visible }\end{array}$ & $\begin{array}{l}\text { Not clearly } \\
\text { visible }\end{array}$ & Reticulate & Reticulate \\
\hline Mural nature & Long parallel & $\begin{array}{l}\text { Long } \\
\text { parallel }\end{array}$ & Long parallel & Long parallel & Long parallel & Long parallel \\
\hline Mural height & $\begin{array}{l}\text { Distinctly } \\
\text { raised }\end{array}$ & $\begin{array}{l}\text { Distinctly } \\
\text { raised }\end{array}$ & $\begin{array}{l}\text { Distinctly } \\
\text { raised }\end{array}$ & Less raised & Distinctly raised & $\begin{array}{l}\text { Distinctly } \\
\text { raised }\end{array}$ \\
\hline Mural length & Long & Long & Long & Long & Long & Long \\
\hline $\begin{array}{l}\text { Mural } \\
\text { alignment }\end{array}$ & Long parallel & $\begin{array}{l}\text { Long } \\
\text { parallel }\end{array}$ & Long parallel & Long parallel & Long parallel & Long parallel \\
\hline
\end{tabular}




\begin{tabular}{|c|c|c|c|c|c|c|}
\hline Characters & $\begin{array}{l}\text { B. x candida } \\
\text { 'Charleston' }\end{array}$ & $\begin{array}{l}\text { B. cubensis } \\
\text { 'Dr.Seuss' }\end{array}$ & $\begin{array}{l}\text { B. sanguinea } \\
\text { 'MishaTora' }\end{array}$ & $\begin{array}{l}\text { B. sanguinea } \\
\text { 'Oroverde' }\end{array}$ & $\begin{array}{l}\text { B. suaveolens } \\
\text { 'Remembrance' }\end{array}$ & $\begin{array}{l}\text { B. suaveolens } \\
\text { 'Valley White' }\end{array}$ \\
\hline Mural width & Broad & Broad & Broad & Narrow & Less broad & Broad \\
\hline Mural packing & $\begin{array}{l}\text { Closely } \\
\text { packed }\end{array}$ & $\begin{array}{l}\text { Closely } \\
\text { packed }\end{array}$ & Closely packed & $\begin{array}{l}\text { Very closely } \\
\text { packed }\end{array}$ & $\begin{array}{l}\text { Less closely } \\
\text { packed }\end{array}$ & $\begin{array}{l}\text { Less closely } \\
\text { packed }\end{array}$ \\
\hline $\begin{array}{l}\text { Mural subunit } \\
\text { nature }\end{array}$ & Cylindrical & Cylindrical & Cylindrical & Cylindrical & Cylindrical & Cylindrical \\
\hline $\begin{array}{l}\text { Mural subunit } \\
\text { alignment }\end{array}$ & $\begin{array}{l}\text { Transverse, } \\
\text { perpendicula } \\
\mathrm{r} \text { to muri }\end{array}$ & $\begin{array}{l}\text { Transverse, } \\
\text { perpendicul } \\
\text { ar to muri }\end{array}$ & $\begin{array}{l}\text { Transverse, } \\
\text { perpendicular } \\
\text { to or diagonal; } \\
\text { sometimes } \\
\text { longitudinal, } \\
\text { parallel to muri }\end{array}$ & $\begin{array}{l}\text { Transverse, } \\
\text { diagonally } \\
\text { oriented }\end{array}$ & $\begin{array}{l}\text { Transverse, } \\
\text { perpendicular to } \\
\text { muri }\end{array}$ & $\begin{array}{l}\text { Transverse, } \\
\text { perpendicular } \\
\text { to muri }\end{array}$ \\
\hline $\begin{array}{l}\text { Mural subunit } \\
\text { thickness }\end{array}$ & Very thin & Very thin & Thin & Thin & Thin & Very thin \\
\hline $\begin{array}{l}\text { Mural subunit } \\
\text { distinctiveness }\end{array}$ & Less distinct & $\begin{array}{l}\text { Less } \\
\text { distinct }\end{array}$ & Distinct & Distinct & Distinct & Distinct \\
\hline $\begin{array}{l}\text { Mural subunit } \\
\text { packing }\end{array}$ & $\begin{array}{l}\text { Closely } \\
\text { packed }\end{array}$ & $\begin{array}{l}\text { Closely } \\
\text { packed }\end{array}$ & Closely packed & $\begin{array}{l}\text { Closely } \\
\text { packed }\end{array}$ & Closely packed & Closely packed \\
\hline $\begin{array}{l}\text { Microscabrae } \\
\text { presence on } \\
\text { muri }\end{array}$ & Present & Present & Present & Present & Present & Present \\
\hline $\begin{array}{l}\text { Microscabrae } \\
\text { density }\end{array}$ & Dense & Dense & Dense & Dense & Dense & Dense \\
\hline $\begin{array}{l}\text { Microscabrae } \\
\text { rows when } \\
\text { present }\end{array}$ & $\begin{array}{l}\text { Single/occasi } \\
\text { onally two or } \\
\text { more }\end{array}$ & $\begin{array}{l}\text { Single/occa } \\
\text { sionally two } \\
\text { or more }\end{array}$ & $\begin{array}{l}\text { Single/occasion } \\
\text { ally two or } \\
\text { more }\end{array}$ & $\begin{array}{l}\text { Single/occasio } \\
\text { nally two or } \\
\text { more }\end{array}$ & $\begin{array}{l}\text { Single/occasiona } \\
\text { lly two or more }\end{array}$ & $\begin{array}{l}\text { Single/occasion } \\
\text { ally two or } \\
\text { more }\end{array}$ \\
\hline $\begin{array}{l}\text { Microscabrae } \\
\text { size }\end{array}$ & Small & Large & Large & Small & Large & Large \\
\hline $\begin{array}{l}\text { Microscabrae } \\
\text { shape }\end{array}$ & Nodular & Nodular & Nodular & Nodular & Nodular & Nodular \\
\hline
\end{tabular}

Table 3:-Pollen morphological characters in species of Datura L. collectedfrom Kerala and Tamil Nadu

\begin{tabular}{|c|c|c|c|c|c|}
\hline Characters & D. innoxia & $\begin{array}{c}\text { D. metel } \\
\text { var. fastuosa }\end{array}$ & $\begin{array}{c}\text { D. metel } \\
\text { var. metel }\end{array}$ & $\begin{array}{c}\text { D. metel } \\
\text { var. rubra }\end{array}$ & D. stramonium \\
\hline \multicolumn{6}{|c|}{ Quantitative characters } \\
\hline $\begin{array}{l}\text { Polar diameter - P } \\
(\mu \mathrm{m})\end{array}$ & $46.80 \pm 1.55$ & $33.60 \pm 3.69$ & $33.00 \pm 3.46$ & $37.20 \pm 1.55$ & $30.60 \pm 4.20$ \\
\hline $\begin{array}{l}\text { Equatorial diam.- } \mathrm{E} \\
(\mu \mathrm{m})\end{array}$ & $50.40 \pm 3.69$ & $25.80 \pm 1.55$ & $32.10 \pm 3.48$ & $30.00 \pm 2.00$ & $46.20 \pm 1.55$ \\
\hline $\mathrm{P} / \mathrm{E}$ & $0.93 \pm 0.07$ & $1.31 \pm 0.22$ & $1.03 \pm 0.05$ & $1.24 \pm 0.06$ & $0.66 \pm 0.10$ \\
\hline Exine thickness $(\mu \mathrm{m})$ & $6.00 \pm 2.00$ & $3.60 \pm 1.27$ & $3.60 \pm 1.27$ & $3.60 \pm 1.27$ & $4.50 \pm 1.58$ \\
\hline Aperture number & 3 & 3 & 3 & 3 & 3 \\
\hline \multicolumn{6}{|c|}{ Qualitative characters } \\
\hline Pollen type & 3-zonocolporate & 3-zonocolporate & 3-zonocolporate & 3-zonocolporate & 3-zonocolporate \\
\hline Pollen size-class & \begin{tabular}{|l|} 
Large \\
\end{tabular} & Medium & Medium & Medium & Medium \\
\hline Characters & D. innoxia & $\begin{array}{c}\text { D. metel } \\
\text { var. fastuosa }\end{array}$ & $\begin{array}{c}\text { D. metel } \\
\text { var. metel }\end{array}$ & $\begin{array}{c}\text { D. metel } \\
\text { var. rubra }\end{array}$ & D. stramonium \\
\hline $\begin{array}{l}\text { Acolpate / } \\
\text { monocolpate grain } \\
\text { presence }\end{array}$ & Absent & Present & Present & Present & Absent \\
\hline Polar outline & $\begin{array}{l}\text { Rounded with } \\
\text { apertures in the } \\
\text { obtuse angles }\end{array}$ & $\begin{array}{l}\text { Rounded with } \\
\text { apertures in the } \\
\text { obtuse angles }\end{array}$ & $\begin{array}{l}\text { Rounded with } \\
\text { apertures in the } \\
\text { obtuse angles }\end{array}$ & $\begin{array}{l}\text { Rounded with } \\
\text { apertures in the } \\
\text { obtuse angles }\end{array}$ & $\begin{array}{l}\text { Rounded with } \\
\text { apertures in the } \\
\text { obtuse angles }\end{array}$ \\
\hline Equatorial outline & Rounded/elliptic & Rounded/elliptic & Rounded/elliptic & Rounded/elliptic & Rounded/elliptic \\
\hline
\end{tabular}




\begin{tabular}{|c|c|c|c|c|c|}
\hline Pollen shape & $\begin{array}{l}\text { Oblate - } \\
\text { spheroidal }\end{array}$ & Subprolate & $\begin{array}{l}\text { Prolate- } \\
\text { spheroidal }\end{array}$ & Subprolate & Oblate \\
\hline $\begin{array}{l}\text { Exine thickness in } \\
\text { optical cross section }\end{array}$ & Thick/thin & Thin & Thin & Thin & Thick \\
\hline $\begin{array}{l}\text { Endocingulum } \\
\text { presence }\end{array}$ & Present & Present & Present & Present & Present \\
\hline Exine ornamentation & Striato-reticulate & Striato-rugulate & Striato-reticulate & Striato-reticulate & $\begin{array}{l}\text { Rugulate- } \\
\text { reticulate }\end{array}$ \\
\hline $\begin{array}{l}\text { Tectum at } \\
\text { mesocolpium }\end{array}$ & Striato-reticulate & Striato-rugulate & Striato-reticulate & Striato-reticulate & $\begin{array}{l}\text { Rugulate- } \\
\text { reticulate }\end{array}$ \\
\hline $\begin{array}{l}\text { Tectum at } \\
\text { apocolpium }\end{array}$ & Rugulate & $\begin{array}{l}\text { Rugulate- } \\
\text { reticulate }\end{array}$ & $\begin{array}{l}\text { Rugulate- } \\
\text { reticulate }\end{array}$ & $\begin{array}{l}\text { Rugulate- } \\
\text { reticulate }\end{array}$ & $\begin{array}{l}\text { Rugulate- } \\
\text { reticulate }\end{array}$ \\
\hline Lumina nature & Reticulate & Granular & Reticulate & Reticulate & Reticulate \\
\hline Mural nature & Long parallel & Long parallel & Long parallel & Long parallel & Short irregular \\
\hline Mural height & Distinctly raised & Distinctly raised & Distinctly raised & Distinctly raised & Distinctly raised \\
\hline Mural length & Long & Long & Long & Long & Short \\
\hline Mural alignment & Long parallel & Long parallel & Long parallel & Long parallel & Short irregular \\
\hline Mural width & Less broad & Less broad & Less broad & Less broad & Less broad \\
\hline Mural packing & $\begin{array}{l}\text { Less closely } \\
\text { packed }\end{array}$ & $\begin{array}{l}\text { Less closely } \\
\text { packed }\end{array}$ & $\begin{array}{l}\text { Less closely } \\
\text { packed }\end{array}$ & $\begin{array}{l}\text { Less closely } \\
\text { packed }\end{array}$ & Loosely packed \\
\hline Mural subunit nature & Striate & Striate & Striate & Striate & Striate \\
\hline $\begin{array}{l}\text { Mural subunits } \\
\text { alignment }\end{array}$ & $\begin{array}{l}\text { Longitudinal, } \\
\text { parallel to muri }\end{array}$ & $\begin{array}{l}\text { Longitudinal, } \\
\text { parallel to muri }\end{array}$ & $\begin{array}{l}\text { Longitudinal, } \\
\text { parallel to muri }\end{array}$ & $\begin{array}{l}\text { Longitudinal, } \\
\text { parallel to muri }\end{array}$ & $\begin{array}{l}\text { Longitudinal, } \\
\text { parallel to muri }\end{array}$ \\
\hline $\begin{array}{l}\text { Mural subunit } \\
\text { thickness }\end{array}$ & Thin & $\begin{array}{l}\text { Thin when } \\
\text { present }\end{array}$ & $\begin{array}{l}\text { Thin when } \\
\text { present }\end{array}$ & $\begin{array}{l}\text { Thin when } \\
\text { present }\end{array}$ & Thin \\
\hline $\begin{array}{l}\text { Mural subunit } \\
\text { distinctiveness }\end{array}$ & Distinct & Very feeble & Very feeble & Very feeble & Distinct \\
\hline $\begin{array}{l}\text { Mural subunit } \\
\text { packing }\end{array}$ & Closely packed & $\begin{array}{l}\text { Not clearly } \\
\text { visible }\end{array}$ & $\begin{array}{l}\text { Not clearly } \\
\text { visible }\end{array}$ & Not clearly visible & Closely packed \\
\hline $\begin{array}{l}\text { Microscabrae } \\
\text { presence on muri }\end{array}$ & Present & Present & Present & Present & Present \\
\hline Microscabrae density & Sparse & Sparse & Sparse & Sparse & Sparse \\
\hline $\begin{array}{l}\text { Microscabrae rows, } \\
\text { when present }\end{array}$ & $\begin{array}{l}\text { Irregularly } \\
\text { scattered }\end{array}$ & $\begin{array}{l}\text { Irregularly } \\
\text { scattered }\end{array}$ & $\begin{array}{l}\text { Irregularly } \\
\text { scattered }\end{array}$ & $\begin{array}{l}\text { Irregularly } \\
\text { scattered }\end{array}$ & $\begin{array}{l}\text { Irregularly } \\
\text { scattered }\end{array}$ \\
\hline Micr-scabrae size & Very small & Very small & Very small & Very small & Very small \\
\hline Microscabrae shape & Granular & Granular & Granular & Granular & Granular \\
\hline
\end{tabular}

The members of Brugmansia had striateor striato-reticulate exines, the latter observed only in the two varieties of $B$. suaveolens (Figs.10 \& 12). The exine was striato-reticulate inDatura except for striato-rugulate pattern in $D$. metel var. fastuosa and rugulate-reticulate exine inD. stramonium. The tectum showed slight variation in exine ornamentation at the apocolpium in some members, eg.rugulatein two members of Brugmansiaandrugulatereticulate in all five taxa ofDatura studied.

The lumina were not visible in the striate pollenof Brugmansiadue to the close packing of the long parallel muri (Fig.2), except inthe two varieties of $B$. suaveolenswhere it was reticulate. In Datura, the lumina were mostly reticulate (Fig.14), except for the granular lumina in D. metel var. fastuosa (Fig.17). B. suaveolenswas similar to Datura in pollen exine thickness, ornamentation and lumina nature (Tables $2 \& 3$ ).

The long muri or liraewere parallelly aligned and distinctly raised in mostmembers (Figs.6 \&8), being broaderand more closely packed in Brugmansia.InD. stramonium, they were short, irregular and loosely packed (Figs.19 \& 23). The mural subunits were distinct or less distinct, thin or very thin, cylindrical, transverse and closely packed, perpendicular to the muri in Brugmansia(Fig. 3). In B. sanguinea, they were occasionally diagonally and/or vertically oriented (Figs.7\& 9). On the other hand, the subunits were striate, thin and longitudinally arranged, parallel to the muri in Datura (Fig. 15), sometimes very feeble and not clearly visible as in D. metel(Figs. 17\& 21). Large and nodular microscabrae were denselyarranged in single or occasionally two or more rows inBrugmansia, in contrast to the small granular ones in Datura which were sparsely present and irregularly scattered (Figs. 7 \&21). 

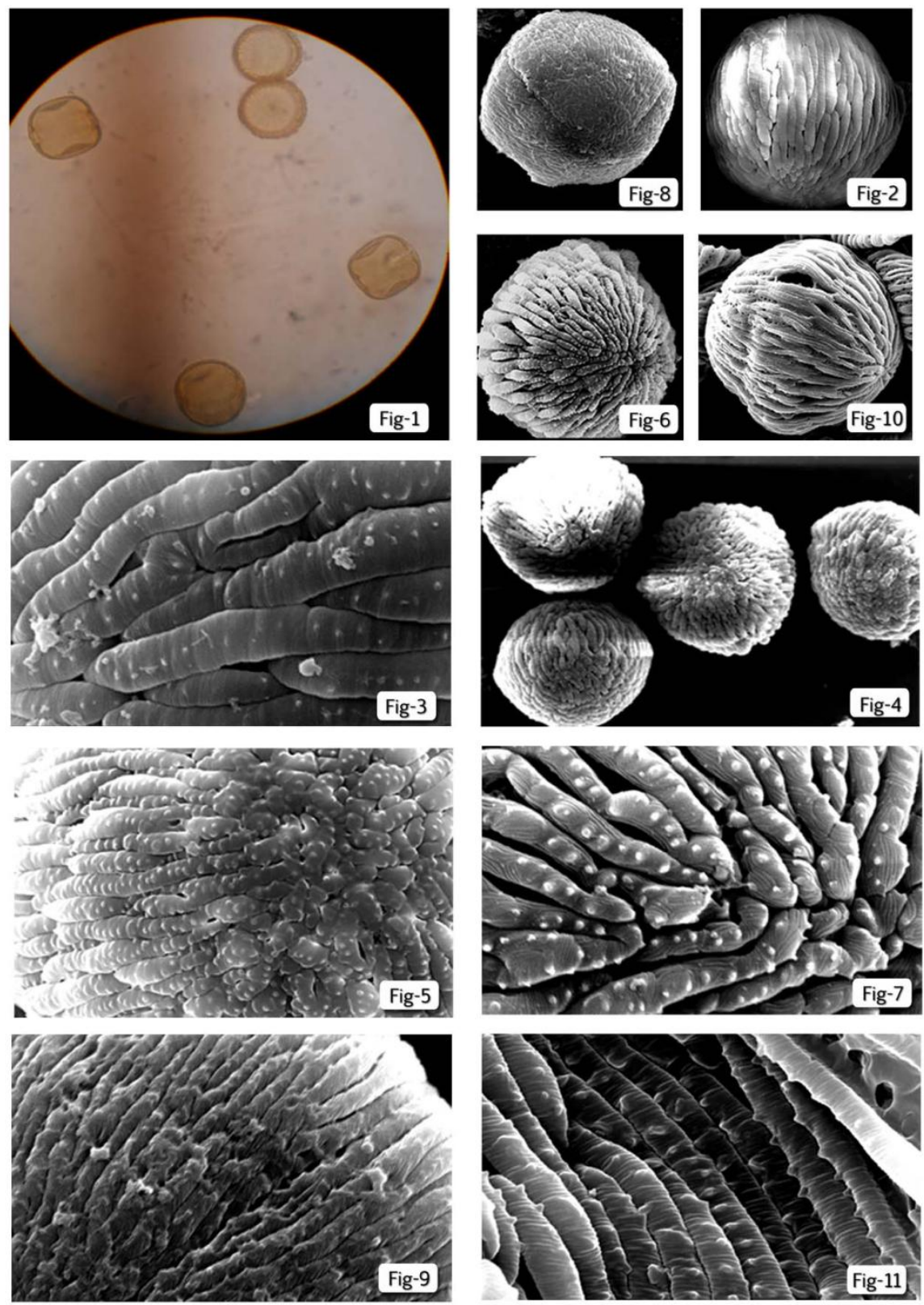

Figs.1-3: Brugmansia x candida 'Charleston' pollen- 40x, 3000x, 10,000x; Figs.4-5: B. x cubensis 'Dr.Seuss' pollen-3000x, 10,000x; Figs.6-7: $B$. sanguinea 'Misha Tora' pollen- 2500x, 10,000x; Figs. 8-9: B. sanguinea 'Oroverde' pollen - 3000x, 10,000x; Figs.10-11: B. suaveolens 'Remembrance' pollen- $1700 x, 10,000 x$. 

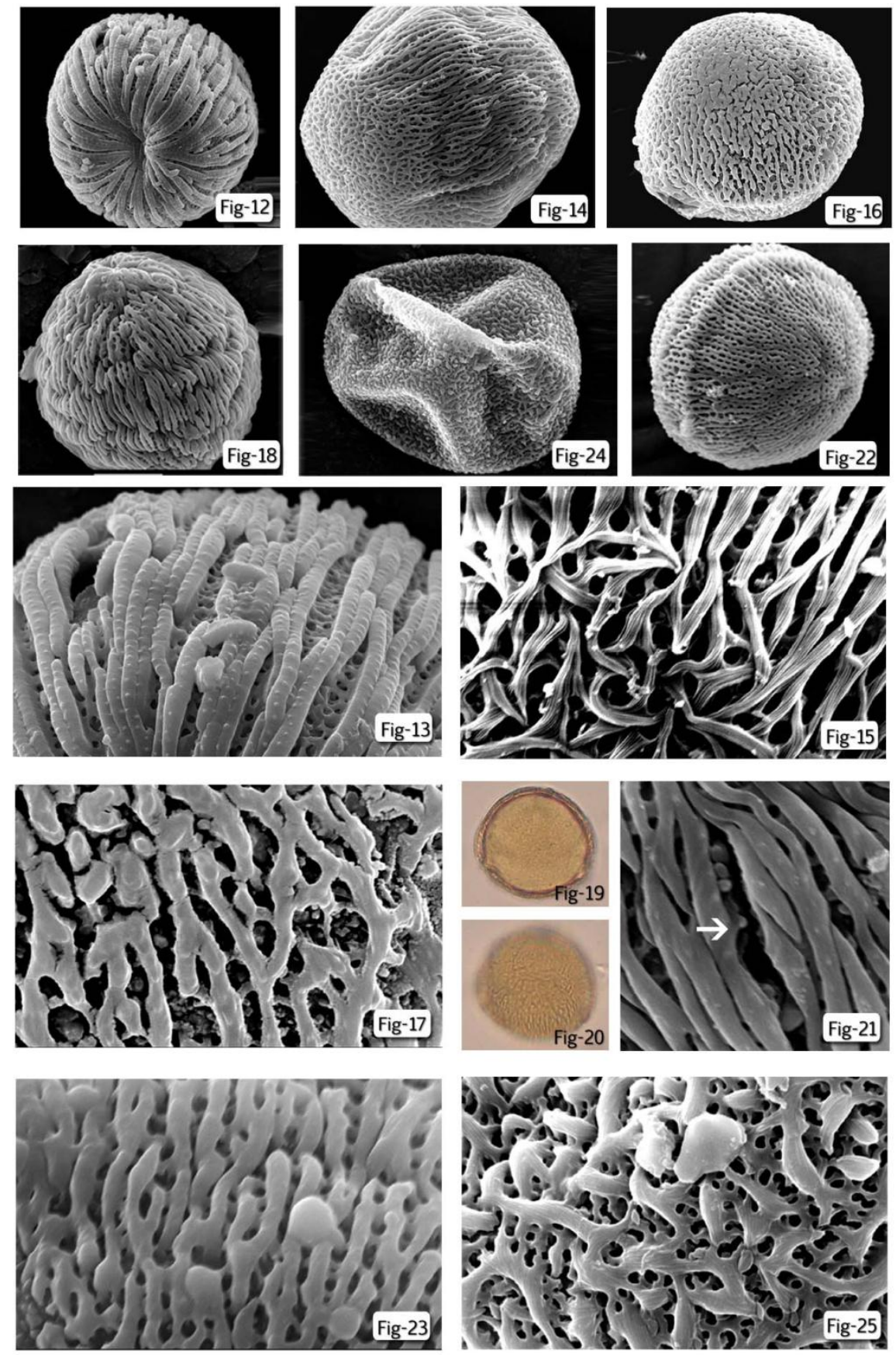

Figs.12-13: B. suaveolens 'Valley White' pollen- 1900x, 5,000x. Figs.14-15: Datura inoxia pollen-5000x, 10, 000x; Figs.16-17: D. metel var. fastuosa pollen-2000x, 10,000x; Figs.18-21: D. metel var. metel pollen2500x, 40x, 40x, 10,000x; Figs.22-23: D. metel var. rubra pollen-5000x, 10,000x; Figs. 24-25:D. stramonium pollen-1500x, 10,000x.

The most extensive pollen morphological study comparing Brugmansia and Datura was made by Knapp and Persoon (1999). They recognized two pollen types on the basis of the thickness of exine, height of muri and alignment of mural subunits viz. Datura inoxia type and Brugmansia sanguinea type. Here the major focus seems to be on the alignment of the mural subunits, a feature distinct to each group being transverse in Brugmansia and longitudinal in Datura (Figs. 3 \&15). But in the present study, B. sanguinea 'MishaTora' showed a combination of both longitudinal and transverse alignment of mural subunits occasionally, even though the major pattern was the transverse type characteristic of the Brugmansia type (Fig.7). Similarly, B. sanguinea 'Oroverde' showed diagonally 
oriented mural subunits (Fig.9). Hence mural subunit alignment cannot be considered aunique or major distinguishing character between the two genera. Moreover,Persoonetal.observed microscabrae in only some species of both genera and did not consider them significant in distinguishing between the two groups. But in the present study, microscabrae were present in all the eleven members and showed clear distinction in their size, form, occurrence and distribution between the two genera. They were large, nodular and dense in single or more rows in Brugmansia in contrast to being small, granular, sparse and irregularly scattered in members of Datura (Figs 5 \& 21). Interestingly, they did not observe these bodies in the American taxa of B. candida and B. sanguinea, while the Indian taxa showed very distinct large, nodular microscabrae in large numbers on the pollen muri (Figs. $3 \&$ 7). Hence microscabrae may also be considered as distinctive markers for comparison between the two genera.

In the light of the above discussion, the pollen of the two groups may be classified into Brugmansia -type and Datura -type as follows :

\begin{tabular}{|l|l|l|l|}
\hline 1. & Brugmansia-type & $:$ & $\begin{array}{l}\text { Exine thick, striate or striato-reticulate, apocolpium same as } \\
\text { mesocolpium in ornamentation; muri or lirae long and closely packed; } \\
\text { mural subunits cylindrical, distinct and transverse - rarely diagonal } \\
\text { and/or longitudinal; microscabrae large, nodular, dense and arranged } \\
\text { almost regularly in one or occasionally more rows. }\end{array}$ \\
\hline 2. & Datura-type & $:$ & $\begin{array}{l}\text { Exine thin, striato-reticulate or sometimes striato-rugulate or rugulate- } \\
\text { reticulate at mesocolpium, apocolpium rugulate-reticulate; muri long or } \\
\text { sometimes short and less closely or loosely packed; mural subunits } \\
\text { striate, feeble and longitudinal; microscabrae small, granular, sparse } \\
\text { and irregularly scattered. }\end{array}$ \\
\hline
\end{tabular}

\section{Conclusions:-}

The exine sculpturing viewed under the Scanning Electron Microscope opens up a treasure trove of pollen microstructural features. These 'micro' traits are most often specific to plant groups and are increasingly used as 'micro' palynological markers in systematic studies and phylogenetic evaluations. The pollen of Datura and its closely related genus Brugmansia are similar being3-zonocolporate,large or medium andstriateor striato-reticulate, occasionally rugulate. Despite these overall similarities, the two genera have been palynologically distinguished largely based on the transverse or longitudinal alignment of the mural subunits in the exine. But longitudinal and/or diagonal orientation of mural subunits have been observed in two varieties of $B$. sanguineapresently studied. Hence mural subunit alignment cannot be depended upon as a unique distinguishing character between pollen of the two genera.In addition, microscabrae on the muri also showed distinction between the two genera, being large, nodular and dense in single or more rows in Brugmansia and small, granular, sparse and irregularly scattered in members of Datura. On the basis of the present observations, two pollen types are proposed here for the two genera studied viz. Brugmansia -type and Datura-type.

\section{Acknowledgements}

The authors are thankful to the University of Kerala for financial support and the Principals, Sree Narayana Colleges, Kollam and Chempazhanthy for facilities provided.

\section{References:-}

1. Adedeji,O. \&Akinniyi, T.A. 2015.Pollen morphology of some species in the family Solanaceae.J. Adv. Lab. Res. Biol.VI (6): 125-129.

2. Al-Quran, S. 2004.Pollen morphology ofSolanaceae in Jordan.Pak. J. Biol. Sci.7 (9) : 1586-1593.

3. Erdtman G. 1952. Pollen Morphology and Plant Taxonomy of Angiosperms.Almquist and Wiksell, Stockholm.

4. Erdtman G. 1966. Pollen Morphology and Plant Taxonomy of Angiosperms.Corrected reprint of the edition of 1952 with a new addendum.Hafner Pub. No:NV.

5. Guang-Fang, P., Shu-Ming, Z., Su-Qin, Z., Dong-Po, Z., Yu-Long, Z. \& An-Ming, L. 1985.Pollen morphology of Chinese Datura and its taxonomic significance.J. Univ. Chinese Acad. Sci.23 (1) : 29-35.

6. Persoon V., Knapp, S. \& Blackmore, S. 1999.Pollen morphology and the phylogenetic analysis of Datura and Brugmansia.In: M. Nee, D.E. Symon, R.N.Lester\& J.P. Jessop (eds.). SolanaceaeIV :Advances in Biology and Utilization. Royal Botanic Gardens, Kew.pp : 171-187. 
7. Lockwood,T.E. 1973. Generic recognition of Brugmansia. Botanical Museum Leaflets (Harvard University)23: 273-284.

8. Mabberley, D.J. 2005. The Plant-Book. A portable dictionary of the Vascular plants. Cambridge: Cambridge University Press.

9. Nair, P.K.K. 1970. Pollen Morphology of Angiosperms. III. Historical and Phylogenetic Study. Vikas Pub. House, Delhi.

10. Perveen, A. \&Qaiser, M. 2007.Pollen morphology offamilySolanaceaefrom Pakistan. Pak. J. Bot., 39 (7) : 2243-2256.

11. Punt W, Blackmore S, Nilsson S \&Le Thomas A. 1994. Glossary of Pollen and Spore Terminology.LPP Contributions Series No.1. LPP Foundation, Uty. Of Utrecht, TheNetherlands.

12. Sasidharan, N. 2004. Biodiversity Documentation for Kerala.Part 6.Flowering Plants.KFRI Handbook No.17. pp. 276-278.

13. Schultes, R.E.\&Raffauf, R.F. 1991. Phytochemical and ethnopharmacological notes on the Solanaceae of the North West Amazon.In:J.G. Hawkes, R.N.Lester, M.Nee\& N. Estrada-R(eds.). SolanaceaeIII: Taxonomy, Chemistry, Evolution. Royal Botanic Gardens, Kew and Linnaean Soc. London.pp - 25-49.

14. Tewari, R.B.\&Nair, P.K.K. 1978. Apertural forms and their evolutionary trends in the pollen grains of Indian Papilionaceae. Ind. J. Bot. 1(1 \& 2): 133-138.

15. Walker, J.W. \& Doyle, J.A. 1975. The basis of angiosperm phylogeny: Palynology. Ann.Miss. Bot. Gard. 62:664-723. 